\title{
Modulación del pensamiento catastrófico, dolor e inflamación en fibromialgia. Del comportamiento a la biologia molecular.
}

\author{
Ana Karen Sandoval Valerio ${ }^{1}$, Víctor Ricardo Aguilera-Sosa ${ }^{1}$, Ángel Miliar García ${ }^{2}$, \\ Modesto Gómez López², Conrado García García ${ }^{3}$, Gerardo Leija Alva ${ }^{1}$, Jennifer V. \\ Sánches-Camacho ${ }^{4,5}$ y Nadia Mabel Péres-Vielma ${ }^{1}$ \\ ${ }^{1}$ Centro Interdisciplinario de Ciencias de la Salud, del Instituto Politécnico Nacional, \\ Ciudad de México, México. \\ ${ }^{2}$ Laboratorio de Biología Molecular. Escuela Superior de Medicina, del Instituto \\ Politécnico Nacional, Ciudad de México, México. \\ ${ }^{3}$ Servicio de Reumatología del Hospital General de México, "Eduardo Liceaga", \\ Ciudad de México, México. \\ ${ }^{4}$ Universidad Estatal del Valle de Ecatepec, Ciudad de México, México. \\ ${ }^{5}$ Sección de Estudios de Posgrado. Escuela Superior de Medicina, del Instituto \\ Politécnico Nacional, Ciudad de México, México.
}

Palabras clave: fibromialgia; intervención cognitivo conductual; IL-6; cortisol; intervención psicofisiológica; pensamiento catastrófico.

Resumen. La fibromialǵia (FM) se caracteriza por la presencia de dolor crónico (DC), estrés y alteraciones cognitivas. La evidencia sugiere que el pensamiento catastrófico (PC) es una constante en esta población, mediado por cortisol, IL-6 y el gen que codifica para el receptor de serotonina 5HT1A (5HT1A). El objetivo de la presente investigación fue identificar si la modificación del PC impacta en estas variables biológicas, para lo cual se compararon los efectos de una intervención cognitivo-conductual (ICC) con los de una intervención psicofisiológica (IPF), en un grupo de mujeres con FM. Fue un estudio no probabilístico, comparativo, experimental y clínico, que tuvo una duración de 11 semanas. Participaron 48 mujeres pacientes del servicio de reumatología del Hospital General de México. Se aplicaron el Cuestionario de Evaluación de la Salud (CES), Escala Catastrófica para Fibromialgia (ECF) y la Escala de Estrés Percibido (EEP). Se cuantificaron la expresión relativa de los genes IL-6 y del receptor de serotonina (5HT1A), mediante PCR en tiempo real, y se determinaron los niveles séricos de IL-6 y cortisol. La ICC produjo cambios significativos en la percepción de estrés $(\mathrm{p}=0,05)$, en PC en la escalas de rumiación $(p=0,05)$, en la magnificación $(p=0,01)$, la desesperanza $(p=0,01)$, el cortisol $(p=0,01)$ y la IL $-6(p=0,01)$; los puntajes de dolor mejoraron en ambos grupos

\footnotetext{
Autor de correspondencia: Nadia Mabel Pérez-Vielma, Sección de Posǵrado e Investigáción, Centro Interdisciplinario de Ciencias de la Salud Unidad Santo Tomás, Instituto Politécnico Nacional. Ciudad de México. México. Tel (52) 5520583053 E-mail:nperezv@ipn.mx
} 
$(\mathrm{p}=0.01)$. La IPF por su parte, mostró un incremento en la expresión relativa del gen que codifica para el receptor de serotonina 5 HT1A $(p=, 05)$. En el grupo de ICC se encontró que la IL-6 es una variable predictora del pensamiento de rumiación. Los resultados permiten concluir que la ICC tuvo mayor impacto en el PC, el estrés, la IL-6 y el cortisol, en comparación con la IPF.

\title{
Modulation of catastrophic thinking, pain and inflammation in fibromyalgia: from behavior to molecular biology.
}

\section{Invest Clin 2021; 62 (2): 140-158}

Key words: fibromyalgia; cognitive behavioral intervention; IL-6; cortisol; psychophysiological intervention; catastrophic thinking.

\begin{abstract}
Fibromyalgia (FM) is a disease characterized by chronic pain (CP), stress, and cognitive alterations. Catastrophic thinking (CT) is mediated by cortisol, IL-6, and the serotonin receptor 5HT1A (5HT1A). The study's objective was to identify whether the modification of CT affects these biological variables, comparing a cognitive behavioral intervention (CBI) and a psychophysiological intervention (PPI) in women with FM. The duration of both interventions was 11 weeks. It was a non-probabilistic, comparative, experimental, and clinical study. Forty-eight women of the Rheumatology Service of the General Hospital of Mexico participated. Health Assessment Questionnaire (HAQDI), Catastrophizing Scale for Fibromyalgia (PCS), and Perceived Stress Scale (PSS) were administered. The relative expression of IL-6 and serotonin receptor (5HT1A) genes were quantified by real-time PCR; likewise, IL-6 and cortisol levels were measured in serum. The ICC showed significant changes in perception of stress $(p=0.05)$, in PC in the rumination scales $(p=0.05)$, magnification $(p=0.01)$, hopelessness $(p=0.01)$, cortisol $(p=0.01)$ and IL-6 $(p=0.01)$. Pain scores $(\mathrm{p}=0.01)$ improved in both groups. The IPF showed an increase in the relative expression of the gene that codes for the serotonin receptor 5HT1A $(p=0.05)$. IL-6 was found to be a predictor of rumination thinking in the ICG group. The results allow us to conclude that ICC had a greater impact on CP, stress, IL-6 and cortisol, compared to the PPI.
\end{abstract}

Recibido: 30-11-2020 Aceptado: 03-04-2021

\section{INTRODUCCIÓN}

La etiología de la fibromialgia (FM) es desconocida; sin embargo, se ha encontrado una desregulación del sistema periférico (alteración del procesamiento del dolor), relacionada con una disfunción en la respuesta al estrés, una reducción en las concentraciones de cortisol, serotonina y de citocinas pro-inflamatorias como las IL-6 e IL-8 (1-6). Por otra parte, se sabe que el dolor y el estrés interactúan con los procesos cognitivos, dando como resultado pensamientos negativos, percepciones distorsionadas, expectativas altas y perfeccionismo, lo que genera un sistema de retroalimentación negativa entre cognnición, estrés y dolor $(7,8)$. 
Las personas con dolor crónico (DC) suelen presentar pensamiento catastrófico (PC) ante experiencias negativas $(9,10), y$ esto provoca el desequilibrio en la inhibición del procesamiento del dolor (11-13).

Lazaridou y col. reportaron que el PC ocasiona el aumento de los niveles plasmáticos y/o séricos de las citocinas proinflamatorias IL-6 e IL-8 (10, 14-16). También, existe evidencia de alteraciones en los valores de cortisol (17); estos cambios funcionales incluyen reactividad reducida del eje hipotálamo-hipófisis-suprarrenal ante el estrés (cortisol), aumento de los niveles de citocinas proinflamatorias, así como disfunción dopaminérgica y serotoninérgica $(18,19)$. Por otro lado, el receptor de serotonina (5HT1A), desempeña un papel clave en la neurotransmisión serotoninérgica; por esta razón, ha sido marcado como un factor etiológico de FM, correlativo con depresión, ansiedad y fatiga (20).

Se ha demostrado que la intervención cognitivo conductual (ICC), resulta eficaz en la modificación del PC y del estrés y, en consecuencia, en la percepción de dolor $(21,22)$. Por otra parte, la intervención psicofisiológica (IPF) busca modificar variables fisiológicas rítmicas que disminuyan el dolor y el estrés $(23,24)$.

En resumen, este estudio tuvo como objetivo evaluar el impacto de la ICC y la IPF en el PC, el estrés percibido, el dolor, los niveles séricos de cortisol e IL-6, así como la expresión génica de la IL-6 y del receptor de serotinina 5HT1A en mujeres con FM, para determinar qué tipo de intervención es más eficaz para modular la expresión de dichos genes.

\section{Construcción de Intervenciones}

El proceso para seleccionar la intervención más eficaz partió de la búsqueda de estudios clínicos y revisiones sistemáticas, especializados en la aplicación de enfoques psicológicos efectivos para reducir el dolor y el estrés en la FM. Se encontró que el 60\% de los estudios consultados utilizó la ICC, al considerarse el tratamiento no farmacológico más efectivo $(22,25)$. En todos los casos la ICC forma parte de tratamientos multicomponentes para el manejo de los síntomas $(26,27)$. Así mismo, existe evidencia en investigaciones como la de Lumley y col. (28) y McCrae y col. (29), que muestran resultados positivos en la mejoría de los síntomas asociados a FM con este abordaje.

A partir de la primera revisión y la etiopatogenia de la FM, se elaboró una segunda intervención que se centró en el control de procesos fisiológicos relacionados con el dolor y el estrés, a través de técnicas para la relajación, debido a la probable correlación entre variables biológicas y psicológicas del estrés. La intervención psicofisiológica es una técnica poco utilizada en comparación con el enfoque cognitivo conductual. Por lo tanto, se realizó la revisión de literatura que sustentara la aplicación de técnicas de respiración y de expresión socioemocional como parte de una segunda intervención; con el objetivo de determinar la eficacia entre ambas intervenciones.

La teoría polivagal y la disautonomía, explican la relación entre las variables biológicas y psicológicas del estrés. La primera especifica que el control vagal cardíaco facilita el comportamiento social a través de estados fisiológicos apropiados, y es clave del comportamiento autorregulador (30). La disautonomía se refiere a una alteración del sistema nervioso autónomo, que en pacientes con FM induce un desequilibrio en su funcionamiento, caracterizado por la hiperactividad simpática en situaciones de estrés que podría estar relacionada con la sintomatología física y psicológica $(31,32)$. En esencia en ambas teorías, esta alteración presenta un desbalance en el tono simpático vagal, que es el que regula automáticamente funciones como la respiración (33).

La revisión sistemática realizada por Jafari y col. concluye que el dolor posiblemente influye en el aumento de la respiración. La técnica de respiración lenta-acelerada se asocia a la reducción del dolor en algunos de 
los estudios; sin embarǵo, no existe evidencia que muestre los mecanismos fisiológicos subyacentes (34). El estudio de Katzman y col. sugiere que el entrenamiento respiratorio ayuda a la reducción de síntomas corporales en la FM (35). Por su parte, Busch y col. encontraron mejoras en la reducción de tensión, ira y síntomas depresivos (36). Finalmente el estudio de Tomas-Carus sugiere que la intervención con entrenamiento respiratorio mejora el dormir (37).

Con base en la revisión de la literatura, nuestro equipo, formado por expertos en los enfoques cognitivo conductual, psicofisiológico y un médico reumatólogo con experiencia en atención a la población objetivo, diseñó ambas intervenciones; desde la estructura de los contenidos, el tiempo necesario para cada sesión, el número de estas y el espacio físico para desarrollarlas. La modulación del pensamiento catastrófico y la reducción del dolor a nivel psicológico y biológico fueron los principales objetivos de las intervenciones.

\section{MATERIAL Y MÉTODOS}

\section{Diseño}

Se trató de un estudio experimental, comparativo, cuantitativo, aleatorio y clínico. La fase basal se refiere a la evaluación previa a las intervenciones, mientras que la fase final se refiere a la evaluación posterior a la última sesión.

\section{Intervenciones}

Ambas intervenciones se dividieron en tres niveles, de acuerdo con las necesidades psicológicas y clínicas descritas en la literatura: 1) psicoeducación, 2) descripción e identificación y 3) nivel explicativo-modificación (Fig. 1).
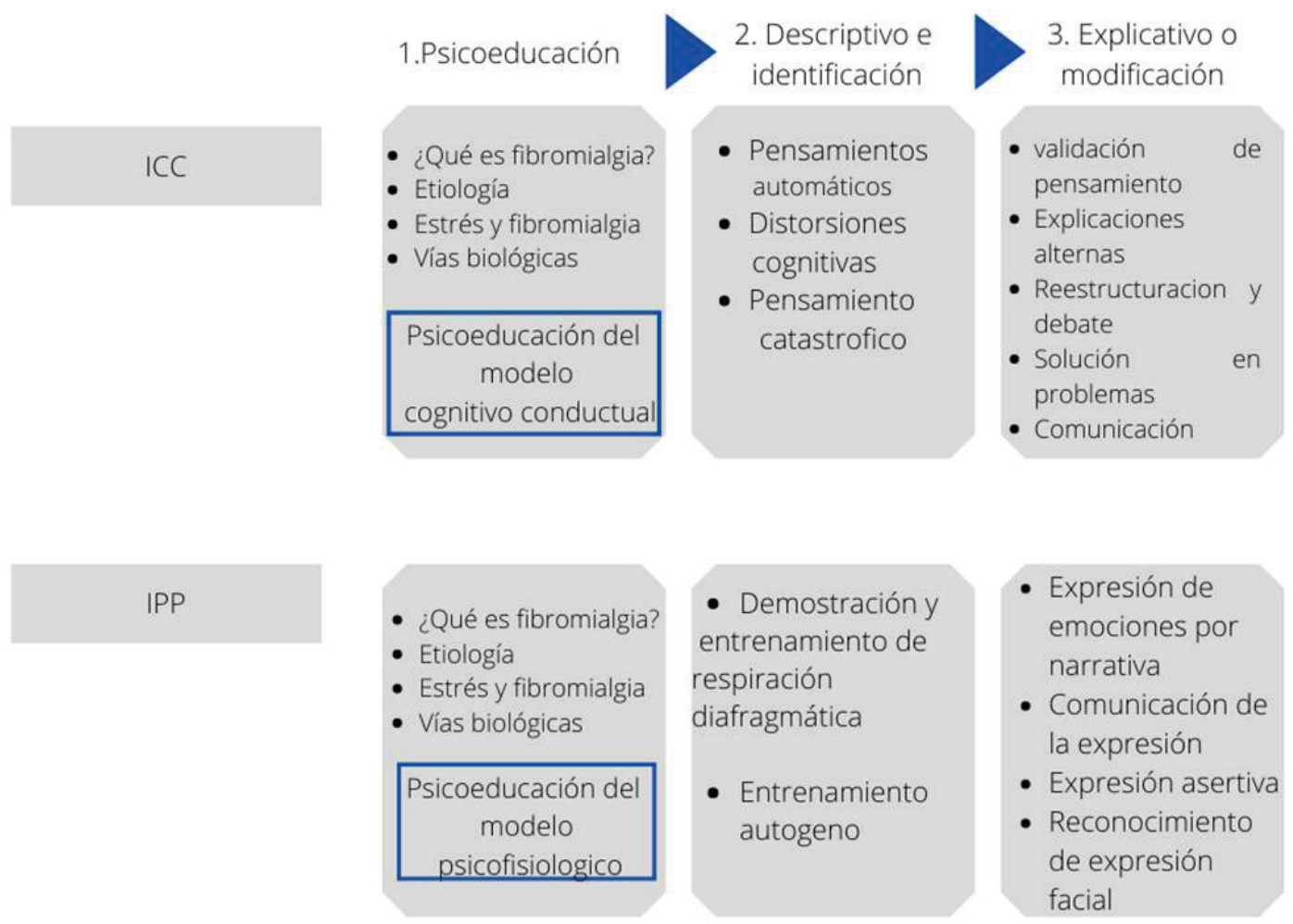

Fig. 1. Estructura de intervenciones. La implementación de los tres niveles tuvo el objetivo de que las participantes tuvieran una estructura óptima para la comprensión de la enfermedad, y comprendieran la relación entre los elementos psicológicos y biológicos, y se familiarizaron con los conceptos, ello como base cognitiva de la modificación del comportamiento del tercer nivel.

Vol. $62(2): 140-158,2021$ 


\section{Variables}

Variable Independiente (VI): Intervenciones psicológicas. Variables Dependientes (VD): Percepción de salud, pensamientos catastróficos, estrés percibido, expresión relativa de los genes de IL-6, del receptor de serotonina 5HT1A y niveles séricos de IL-6 y cortisol.

\section{Reclutamiento}

Se seleccionaron a 48 participantes de la clínica de fibromialgia, las cuales se encontraban activas, de la Unidad de Reumatología del Hospital General de México "Eduardo Liceaga". Todas las participantes contaban con diagnóstico de fibromialgia según los criterios del Colegio Americano de Reumatología (ACR 1990) y tenían alrededor de 6 meses asistiendo a consulta. El proceso de selección fue el siguiente:

Las participantes se reclutaron en la consulta de fibromialǵia, los días jueves durante 3 a 4 meses. Se eligió el jueves por ser el día en que se reciben pacientes que tienen un seguimiento temprano. Posteriormente, se seleccionaron las pacientes que cumplieran con los criterios de inclusión y exclusión. Por último, se les invitó a participar y se les explicó detalladamente en qué consistía la investigación. Dentro de esos meses de reclutamiento se entrevistaron 80 pacientes; de las cuales 51 cumplieron con los criterios de inclusión y 48 finalizaron el estudio.

El perfil terapéutico de cada grupo, se identificó una vez obtenidos los resultados basales, ya que desde un inicio la selección se dio de forma aleatorizada basándose en los criterios de inclusión y exclusión.

Criterios de inclusión: 1. Pacientes de sexo femenino, entre 18 y 65 años. 2. Participación voluntaria. 3. Firma y entrega del consentimiento informado. 4. Sin comorbilidades, en caso de presentar fibromialgia debía ser el padecimiento con mayor exacerbación (por ejemplo, si presentase artritis). 5. Diaǵnóstico emitido por un reumatólogo, ante los criterios del ACR, 1990. 6. Diagnóstico de fibromialgia de aproximadamente 6 meses 7. Tratamiento farmacológico reumatológico estable de al menos seis meses.
Criterios de exclusión: 1. Diaǵnóstico de alguna enfermedad psiquiátrica, basado en datos de entrevista clínica. 2. Diagnóstico no definido que no confirmase el de fibromialgia. 3. Prescripción de fármacos de tipo esteroideo. 4. Prescripción de antidepresivos (inhibidores de la recaptura de 5HT1A).

Criterios de eliminación: 1 . Inasistencia a por lo menos cuatro sesiones. 2. Inasistencia a las evaluaciones (Pre y post estudio). 3. Evaluaciones incompletas.

\section{Intervención Cognitivo Conductual}

En el grupo de ICC participaron 24 mujeres, organizadas en dos grupos de 12 para facilitar el proceso. A cada una de ellas se le proporcionó un manual de intervención con actividades para realizar por sesión y objetivos, elaborado por la investigadora principal y el colaborador experto en ICG (Tabla I).

La ICC constó de 11 sesiones de una hora, una vez por semana. El primer nivel o de psicoeducación tuvo como objetivo la generación de conocimiento acerca de la enfermedad, sus causas, los aspectos fisiológicos y biológicos, lo contextual-familiar y, por último, información sobre la base del modelo de la ICC. El segundo nivel de identificación se enfocó en la detección de productos cognitivos: pensamientos automáticos, distorsiones cognitivas, creencias disfuncionales, actitudes disfuncionales y su correlación con la emoción y la conducta, narrados desde la experiencia de cada participante. Finalmente, en el tercer nivel (explicación y modificación), se buscó que las participantes comenzaran a validar sus pensamientos catastróficos, para cambiarlos por pensamientos adaptativos, pensamientos dirigidos y pensamientos funcionales, mediante la aplicación de las técnicas de:

1) Identificación de pensamientos automáticos, distorsiones, pensamientos catastróficos, creencias y actitudes disfuncionales. 
TABLA I

DESCRIPCIÓN DE SESIONES DE LA INTERVENCIÓN COGNITIVO CONDUCTUAL

\begin{tabular}{ccc} 
No. & Objetivos & Técnica \\
Sesión & & \\
\hline
\end{tabular}

PRIMER NIVEL: PSICOEDUCACIÓN

1 Psicoeducación en FM, etiología, síntomas,

Psicoeducación sistemas involucrados

Realización de objetivos

¿Qué se espera?

\section{SEGUNDO NIVEL: DESCRIPTIVO}

2 Identificar PA, ereencias irracionales y distorsiones cognitivas referentes al dolor.

Identificación

Descripción

Ejercicio ABC

3 Evaluar y analizar respuestas y proceso de PA,

Autoevaluación considerando consecuencias conductuales y emocionales disfuncionales.

Productos informacionales

TERCER NIVEL: EXPLICATIVO

$4 \quad$ Evaluación de sistema de PA para identificar PC

Analizar y examinar pruebas empíricas del PC referente al dolor. Identificar emociones y conductas.

5 Modificación de PC relacionados con el dolor.

Generación de hipótesis

"Método Alto del pensamiento"

6 Cuestionamiento de esquemas ideológicos, valorativos, motivaciones y constructivos.

Capacidades

Actitudes

Pensamiento y afecto

Debate para reestructuración de esquemas.

$7 \quad$ Cambio de "significado" y aceptación del

Conocimiento declarativo y procedimental dolor.

8 Identificar beneficios y repercusiones de cogniciones en FM y dolor.

"Lo que quiere y cree" Procesamiento controlado.

$9 \quad$ Identificar y generar formas de afrontamiento. Revalorar el proceso de solución de problemas.

10 Especificar orientación y actitud hacia problemas.

Alternativas en la toma de decisiones.

Expresión y comunicación asertiva.

$11 \quad$ Cierre

Percepción y control personal.

Reevaluación y revisión de objetivos.

Formas de mantenerlos.

Identificación de sistemas y conclusiones.

Vol. 62(2): 140 - 158, 2021 
2) Explicación alterna.

3) Gráficos circulares (qué situaciones están en su control y pueden modificar y cuáles no).

4) Debates lógicos y empíricos (identificar los beneficios que obtienen de ese pensamiento y comportamiento).

5) Método en solución de problemas (principalmente de comunicación).

En la última sesión, se retroalimentó lo aprendido y las experiencias personales.

\section{Intervención Psicofisiológica}

En el grupo de intervención psicofisiológica (IPF), participaron 24 mujeres, organizadas en dos grupos de 12 para facilitar el proceso. La IPF constó de 11 sesiones realizadas una vez por semana con duración de 1 hora. El nivel psicoeducativo fue construido de la misma forma que la ICG: descripción de principios, definiciones, la relación de fibromialgia con el estrés, lo biológico, lo fisiológico y lo psicológico. De igual forma se brindó psicoeducación sobre el enfoque psicofisiológico y los modelos teóricos que lo sustentan. El segundo nivel tuvo como objetivo que las participantes identificaran la forma en la que respiraban y su relación con la propia respuesta fisiológica y psicológica. Se proporcionó entrenamiento autógeno y de respiración diafragmática; este último con la finalidad de que aprendieran a autogestionar el control de las respuestas fisiológicas con base en la respiración y la relajación. Para el tercer nivel, el de modificación, la indicación fue que el entrenamiento autógeno y de respiración diafragmática fuera practicado en casa, acompañado de las técnicas del tercer nivel:

1) Expresión de emociones a través de la narrativa (para la expresión de aquellas situaciones o eventos que activaban el dolor).

2) Narraciones escritas en tres momentos en primera y tercera persona, y una última donde incluyeran emociones que venían en un listado de respuestas emocionales básicas.
3) Técnica de comunicación no violenta y asertividad (con "role playing" representaron la forma en la que regularmente expresan malestar, enojo, frustración y dolor, ya sea físico o "emocional"); la instrucción fue que escogiieran en la imaǵinación a una persona importante, para expresar emociones, $y$ por aproximaciones sucesivas, se acercaran a la comunicación asertiva.

4) Técnica de reconocimiento de emociones, por medio de la expresión facial.

Por último, se reforzó la generación de nuevas redes de apoyo y de mejoramiento de las actuales. En la última sesión se retroalimentó sobre lo aprendido y las experiencias vividas. A cada participante se le dio un manual de la intervención que contenía actividades que se realizaron por sesión y objetivos, elaborado por la investigadora principal y el experto en el modelo IPF (Tabla II).

\section{Aspectos éticos}

La presente investigación fue aprobada por el Comité de Ética en Investigación del Centro Interdisciplinario de Ciencias de la Salud Unidad Santo Tomás (CEI-CICS-021). Se obtuvo el consentimiento informado por escrito de todas las participantes. El ensayo se llevó a cabo de acuerdo con los principios éticos de la Declaración de Helsinki y fue consistente con las Guías de Buenas Prácticas Clínicas. Esta investigación se consideró con riesgo mínimo con base en el Reglamento de la Ley General de Salud en Materia de Investigación de Salud; Artículo 17 que considera riesgo de la investigación a la probabilidad de que el sujeto sufra algún daño como consecuencia inmediata/tardía.

\section{Terapeutas}

El diseño y construcción de las intervenciones fue dirigido por especialistas en lo cognitivo conductual, y psicofisiológico; todos profesionales en salud mental. La psicóloga clínica que participó en la elaboración de los modelos fue quien implementó ambas intervenciones, debido a su experiencia clínica en FM. 
TABLA II

DESGRIPGIÓN DE SESIONES DE LA INTERVENCIÓN PSICOFISIOLÓGICA.

\begin{tabular}{ccc} 
No. & Objetivos & Téenica \\
Sesión & & \\
\hline
\end{tabular}

PRIMER NIVEL: PSICOEDUCACIÓN

1 Psicoeducación de enfermedad, induciendo al modelo de respiración diafraǵmática.

Psicoeducación

Realización de objetivos

¿Qué se espera?

\section{SEGUNDO NIVEL: DESCRIPTIVO}

2 Explicar los diferentes tipos de respiración. Práctica de respiración diafraǵmática.

3 Exponer importancia sobre el ritmo en respiración diafraǵmática.

Respiración diafraǵmática mediante ejemplos.

Demostración de respiración rítmica.

TERCER NIVEL: EXPLICATIVO

4 Escritura y narración de eventos que causara activación del dolor, y su relación con emociones.

5 expresión asertiva de "lo que quieren comunicar" en relación al dolor.

6 Identificación de su mensaje, narrando en tercera persona.

$7 \quad$ Que las pacientes sean capaces de aprender de manera asertiva a expresarse, ante identificación de escritura.

8 Reconocer emociones principales, expresiones faciales en otros y sí misma.

$9 \quad$ Importancia de redes de apoyo.

10 Reevaluación y revisión de objetivos.

11 Cierre
Se explicará qué es la escritura emocional autorreflexiva. Se realizará el ejercicio uno, previamente se evaluará el nivel de dolor.

Palabras que utilizan positivas, negativas y enlaces para decir lo que piensan y sienten.

Utilizar el pasado como herramienta.

Hojas de registro para la evaluación de frases clave.

Mostrar caras y rostros, que anoten qué emoción es la que corresponde con la imagen.

Anotar con qué redes cuentan y cómo se han mantenido.

Discusión

Percepción y control personal

\section{Evaluación}

La evaluación se realizó en dos momentos: basal (antes de la primera sesión de intervención y final (posterior a la última sesión de intervención). En la etapa basal se citó a las participantes a dos sesiones diferentes para su evaluación; en la primera se hizo la toma de muestra de sangre periférica para la evaluación biológica y molecular y en la segunda se aplicaron los instrumentos psicológicos. Lo anterior se realizó con la finalidad de no interferir la atención de una actividad con otra, así como para evitar el cansancio de las participantes por permanecer sentadas y realizar varias actividades.

La evaluación final, tuvo la misma dinámica que la basal, después de la última

Vol. 62(2): 140 - 158, 2021 
sesión de intervención, se citó una semana después a las participantes para hacer la toma de muestra de sangre. Un día después se realizó la evaluación psicológica, de manera separada para que no hubiese algún sesgo de tiempo y forma.

En ambas etapas, basal y final, la toma de muestra de sangre se realizó a las $8 \mathrm{am}$, esto para determinar el rango normal de los niveles séricos de cortisol, debido a que estos cambian durante el día y la noche.

Únicamente tres participantes no concluyeron el tratamiento, una de ellas, que se encontraba en la intervención psicofisiológica, concluyó pero no realizó la última evaluación, y dos participantes de la intervención cognitivo conductual realizaron la evaluación basal y participaron en cinco sesiones, pero no concluyeron el total de la intervención ni la evaluación final.

\section{Sujetos de estudio y muestras de sangre}

Se trató de un estudio no probabilístico, comparativo, experimental y clínico; ICC $\mathrm{n}=24$ e IPF $\mathrm{n}=24$ del servicio de Reumatología del Hospital General de México Dr. Eduardo Liceaga de la CDMX. La selección de las participantes para cada una de las intervenciones, se realizó de manera aleatorizada por método de tómbola. Se obtuvo una muestra de sangre de vena antecubital del brazo para determinación de mediciones bioquímicas y expresión génica.

\section{Instrumentos}

Se aplicó el Cuestionario de Evaluación de la Salud (CES) validado en la población mexicana, que evalúa la influencia de enfermedades crónicas y la salud en conjunto, con tres escalas:

- Discapacidad: (nivel de habilidad funcional). Escala de 0-3, donde 0 es sin discapacidad y 3 completamente desactivado.

- Dolor: presencia/ausencia de dolor durante la última semana. Escala de 0-3, donde 0 es sin dolor y 3 dolor intenso.
- Estado de salud: ("Termómetro de sensación"). Con base en una línea de $15 \mathrm{~cm}$ en donde 0 es "Muy bien" y 10 "Muy deficiente".

- Se aplicó la Escala Catastrófica para Fibromialgia (ECF) con 13 ítems y 3 factores referentes a catastrofización del dolor (rumiación, aumento e impotencia). Se les pidió a las participantes reflexionar sobre experiencias dolorosas, además de que identificaran los pensamientos automáticos y emociones negativas asociados al nivel de dolor, con la guia subjetiva de 5 puntuaciones, en donde 0 representó "nada de dolor", y el 4, "todo el tiempo tengo dolor".

- Finalmente, se aplicó la Escala de Estrés Percibido (EEP) que evalúa la percepción de estrés durante el último mes; 14 ítems con patrón de respuesta politómica: nunca, casi nunca, de vez en cuando, a menudo y muy a menudo, que dan puntuaciones de cero a cuatro. Los ítems 4, 5, 6, 7, 9, 10 y 13 tienen patrón reverso de cuatro a cero, entre 0 y 56 , a una mayor puntuación corresponde un mayor nivel de estrés percibido.

\section{Expresión Génica y Niveles Séricos de Cortisol e IL-6}

Los niveles séricos de cortisol se cuantificaron mediante quimioluminiscencia, mientras los niveles séricos de IL-6, mediante la técnica de ELISA (Cayman Chemical Item No. 501030, Ann Arbor, MI USA). Así mismo, se extrajo el ARN de muestras de sangre periférica para determinar la expresión génica de IL-6 y 5HT1A, mediante PCR en tiempo real (qPCR), en un termociclador Techne Prime Pro 48. La extracción de ARN se realizó con la técnica de TRIzol (Roche Applied Science, Indianapolis, IN, USA). Se verificó cantidad, pureza e integridad del ARN. Posteriormente, se empleó el estuche de síntesis de ADNc (Roche Diagnostics, GmbH Mannheim, Germany) tomando $1 \mu \mathrm{g}$ de la muestra total de ARN. La transcripción inversa se realizó con random primers siguiendo las instrucciones especificadas por el fabricante, en un termociclador Techne. La concentración de ADNe se determinó con nanoespectrofotómetro, pos- 
teriormente para la qPCR se emplearon oligonucleótidos específicos para los genes IL-6 y 5HT1A, diseñados con el software (https:// qper.proberinder.com/organism.jsp).

La reacción para sintetizar $\mathrm{ADNc}$ se realizó de acuerdo con instrucciones de Roche Diagnostics, GmbH, $1 \mu \mathrm{L}$ de ADNe en dilución apropiada en un termociclador punto final de TECHNE. Posteriormente, la qPCR se efectuó en el PrimePro48 de TECHNE, con condiciones de amplificación: desnaturalización inicial $10 \mathrm{~min}$ a $95^{\circ} \mathrm{C}$, seguida por 45 ciclos de $10 \mathrm{~s}$ a $94^{\circ} \mathrm{C}, 20 \mathrm{~s}$ a $60^{\circ} \mathrm{C}$ y $5 \mathrm{~s}$ a $72^{\circ} \mathrm{C}$. La cuantificación de qPCR se ejecutó por medio de interpolación de valores dentro de curva estándar y a diferentes diluciones exponenciales de concentración inicial del templado. Los datos se normalizaron usando el gen constitutivo $18 \mathrm{~S}$ para determinar diferencias en eficiencia de amplificación y cantidad de templado en cada reacción.

\section{Análisis Estadístico}

Se realizaron ANOVAS de medidas repetidas para identificar las diferencias entre sujetos e intra sujetos, correlaciones de Pearson para identificar las relaciones entre variables, y regresiones lineales (método paso por paso), para identificar variables predictoras $(\mathrm{p}<0,05)$, usando el programa SPSS 24.

\section{RESULTADOS}

A continuación se muestran los datos sociodemográficos de ambos grupos (Tabla III).

\section{Escalas Psicológicas}

En la tabla de ANOVAS, referentes a las escalas psicológicas (Tablas IV y V), se muestra que en ambos grupos la disminución en los puntajes finales en comparación a los basales, en cuanto a dolor, funcionalidad y salud, tuvo la misma eficacia ( $p \geq 0,05)$.

Destacamos que en el instrumento de percepción de estrés, el catastrofismo en sus tres subescalas (rumiación, maǵnificación y desesperanza) fue estadísticamente significativo $(\mathrm{p}=<0,05)$, y potencia cercana a 1 , y favorables al grupo ICC en comparación con el grupo IPF, dado que en la prueba entre grupos mostró mejores resultados, como se observa en la tabla de medidas repetidas (Tabla V).

\section{Expresión Génica y Niveles Séricos de Cortisol e IL-6}

En la tabla de la ANOVA de las variables biológicas (Tablas VI y VII) en ICC observamos una disminución de la expresión génica de IL-6 y del gen del receptor de serotonina (5HT1A), en comparación a IPF, dado que disminuyeron las UER después del tratamiento. Mientras que la expresión génica de ambos genes aumentó en la IPF.

En cuanto a los niveles séricos de cortisol, se observa aumento en la medición final en el grupo ICC, a diferencia del grupo de IPF.

Como se observa en la Tabla VI, se encontraron diferencias significativas $(p=$ $0,05)$ en los niveles séricos de cortisol e IL6 , pero no en los niveles de expresión relativa del gen 5HT1A, dado que los grupos obtuvieron puntuaciones similares. Mientras que IPF mostró aumento en la expresión relativa del gen de IL-6.

\section{TABLA III \\ DATOS SOCIODEMOGRÁFICOS DE AMBOS GRUPOS.}

\begin{tabular}{|c|c|c|}
\hline Factor & ICC & IPF \\
\hline Edad en años & $\begin{array}{c}X(\mathrm{DE}) \\
46,86(12,36)\end{array}$ & $\begin{array}{c}X(\mathrm{DE}) \\
43,35(11,31)\end{array}$ \\
\hline Estado civil & $\%$ & $\%$ \\
\hline Casada & 40,0 & 70,0 \\
\hline Soltera & 31,8 & 25,0 \\
\hline Divorciada & 18,2 & 18,2 \\
\hline Unión libre & 9,1 & 5,0 \\
\hline
\end{tabular}

$\mathrm{ICC}=$ Intervención Cognitivo Conductual; IPF = Intervención Psicofisiológica. 
TABLA IV

ANOVA DE MEDIDAS REPETIDAS DE VARIABLES PSICOLÓGICAS, PRE Y POST INTERVENCIONES.

\begin{tabular}{|c|c|c|}
\hline Variables & $\begin{array}{c}\text { ICG } \\
\mathrm{X}(\mathrm{DE})\end{array}$ & $\begin{array}{c}\text { IPF } \\
X(D E)\end{array}$ \\
\hline Estrés pre & $36,09(4,58)$ & $39,95(3,03)$ \\
\hline Estrés post & $27,36(3,96)^{* * *}$ & $30,45(3,91)^{* * *}$ \\
\hline Catastrofismo Rumiación Pre & $7(5,32)$ & $10,2(3,69)$ \\
\hline Catastrofismo Rumiación Post & $2,23(0,68) \mathrm{NS}$ & $5,4(3,86)^{* * *}$ \\
\hline Catastrofismo Magnnificación Pre & $5,8(4,7)$ & $5,9(3,09)$ \\
\hline Catastrofismo Maǵnificación Post & $2,0(1,95) * * *$ & $4,6(2,67)^{* * *}$ \\
\hline Catastrofismo Desesperanza Pre & $11,32(7,58)$ & $13,2(5,6)$ \\
\hline Catastrofismo Desesperanza Post & $2,7(2,92)^{*}$ & $7,85(5,26) * *$ \\
\hline Funcionalidad Dolor Pre & $8,4(5,44)$ & $10,8(6,38)$ \\
\hline Funcionalidad Dolor Post & $4,3(2,96)^{* * *}$ & $6,85(6,38)^{* * *}$ \\
\hline Nivel de Dolor Pre & $7,3(1,29)$ & $8,20(1,36)$ \\
\hline Nivel de Dolor Post & $4,3(1,39)^{* * * *}$ & $4,7(1,67)^{* * *}$ \\
\hline Salud Pre & $7,3(1,72)$ & $8,0(1,33)$ \\
\hline Salud Post & $4,1(1,58)^{* * *}$ & $5,17(2)$ \\
\hline
\end{tabular}

TABLA V

ANOVAS ENTRE-GRUPOS DE VARIABLES PSICOLÓGICAS ICC CONTRA IPF.

\begin{tabular}{|c|c|c|c|c|}
\hline Variables & $\mathrm{p}$ & $\mathrm{F}$ & $\mathrm{P}$ & Eta Cuadrada \\
\hline Estrés & 0,001 & 122,4 & 0,1 & 122,407 \\
\hline Catastrofismo Rumiación & 0,001 & 15,63 & 0,1 & 0,28 \\
\hline Catastrofismo Magnnificación & 0,001 & 7,23 & 0,74 & 0,15 \\
\hline Catastrofismo Desesperanza & 0,001 & 5,72 & 0,64 & 0,12 \\
\hline Funcionalidad Dolor & 0,29 & 5,1 & 0,00 & 0,05 \\
\hline Nivel de Dolor & 0,64 & 0,21 & 0,074 & 0,005 \\
\hline Salud & 0,04 & 4,57 & 0,54 & 0,10 \\
\hline
\end{tabular}

Estos resultados corroboran que las pacientes mostraron niveles altos de la expresión del gen de IL-6 previo al tratamiento en ambos grupos; sin embargo, en ICC disminuyeron los puntajes finales de la expresión génica, mientras que en IPF aumen- taron. No obstante, este cambio no se reflejó en ningún grupo respecto a los niveles séricos de IL-6. Mientras que el cortisol, se encontró aumentado en ICC con signnificancia estadística, y se halló disminuído en IPF, aunque de manera no significativa. 
TABLA VI

ANOVAS MEDIDAS REPETIDAS DE VARIABLES BIOLÓGICAS, PRE Y POST INTERVENCIONES.

\begin{tabular}{lcc}
\hline Variables & $\begin{array}{c}\text { ICC } \\
\text { Media (DE) }\end{array}$ & $\begin{array}{c}\text { IPF } \\
\text { Media (DE) }\end{array}$ \\
\hline 5HT1A Pre (UER) & $0,33(0,25)$ & $0,13(0,20)$ \\
5HT1A Post (UER) & $0,22(0,25)$ & $0,24(0,21)$ \\
Cortisol Pre sérico (ug/dL) & $8,23(2,97)$ & $8,27(2,46)$ \\
Cortisol Post sérico (ug/dL) & $10,36(3,41)^{* *}$ & $7,43(2,94)$ \\
IL-6 Pre (UER) & $0,19(0,25)$ & $0,28(0,33)$ \\
IL-6 Post (UER) & $0,05(0,09)^{* *}$ & $0,64(0,21)^{* *}$ \\
Elisa IL-6 Pre (pg/mL) & $6,73(5,39)$ & $8,37(9,13)$ \\
Elisa IL-6 Post (pg/mL) & $0,05(0,9)$ & $8,63(9,90)$ \\
\hline
\end{tabular}

ICC $=$ Intervención Cognitiva Conductual, IPF =Intervención Psicofisiológica, $\mathrm{p}={ }^{*}<0,05,{ }^{* *}<0,01$.

TABLA VII

ANOVAS ENTRE-GRUPOS DE VARIABLES BIOLÓGICAS, ICG CONTRA IPF.

\begin{tabular}{lcccc}
\hline Variables & $\mathrm{p}$ & $\mathrm{F}$ & $\mathrm{P}$ & Eta Cuadrada \\
\hline 5HT1A Pre (UER) & 0,038 & 4,61 & 0,55 & 0,10 \\
Cortisol Pre sérico (ug/dL) & 0,02 & 5,25 & 0,60 & 0,11 \\
IL-6 Pre (UER) & 0,001 & 20,97 & 0,99 & 20,97 \\
Elisa IL-6 Pre (pg/mL) & 0,77 & 0,81 & 0,059 & 0,002 \\
\hline
\end{tabular}

$\mathrm{ICC}=$ Intervención Cognitiva Conductual, $\mathrm{IPF}=$ Intervención Psicofisiológica, $\mathrm{p}=$ significancia, $\mathrm{F}=$ variabilidad de medias, $\mathrm{P}=$ Potencia. $\mathrm{UER}=$ unidades de expresión relativa.

\section{Correlaciones de Pearson}

$\mathrm{Al}$ calcular las correlaciones de Pearson, encontramos correlación positiva en el grupo de ICC, entre catastrofismo (rumiación) e IL-6 (expresión génica), a menor catastrofismo, menor es la expresión del gen de IL-6.

Por otro lado, en relación con IPF, se obtuvieron correlaciones positivas con el receptor de serotonina 5HT1A y la percepción de estrés, dolor y salud; a mayor expresión del gen del receptor 5 HT1A, la percepción de estrés, salud y dolor, será también mayor (Figs. 1 y 2).

\section{Regresiones lineales}

El dolor, estrés y el pensamiento catastrófico son las principales variables que se buscó disminuir. Por lo tanto, se realizó un análisis de regresión lineal considerando esas variables como criterio de predicción, así como la expresión génica (Tabla VIII).

La Fig. 2 muestra de forma gráfica los resultados más relevantes de correlaciones y regresión lineal en ICG. En esta intervención se encontró que el estado de salud predice el nivel de dolor y que el catastrofismo (rumiación) predice los niveles séricos de IL-6 (proteína). 


\section{TABLA VIII}

REGRESIONES LINEALES DE ESCALAS PSICOLÓGICAS Y VARIABLES METABÓLICAS.

\begin{tabular}{ccccccc}
\hline Grupo & Variable dependiente & Predictor & $\mathrm{r}$ & $\mathrm{r}^{2}$ & $\mathrm{p}$ & $\beta$ \\
\hline \multirow{2}{*}{ ICC } & Estado de Salud & Nivel de Dolor & 0,828 & 0,685 & 0,0001 & 0,819 \\
& Catastrofismo (Rumiación) & IL-6 expresión del gen & 0,475 & 0,226 & 0,025 & $-0,105$ \\
IPF & Estado de Salud & Nivel de Dolor & 0,802 & 0,643 & 0,0001 & 0,472 \\
& Funcionalidad & Nivel de Dolor & 0,867 & 0,752 & 0,0001 & 0,148 \\
& Estado de Salud & Receptor 5HT1A & 0,612 & 0,375 & 0,005 & 0,346 \\
\hline
\end{tabular}

$\mathrm{r}=$ correlación, $\mathrm{r}^{2}=\mathrm{r}$ cuadrada, $\mathrm{p}=$ significancia, $\beta=$ coeficiente de regresión estandarizado.

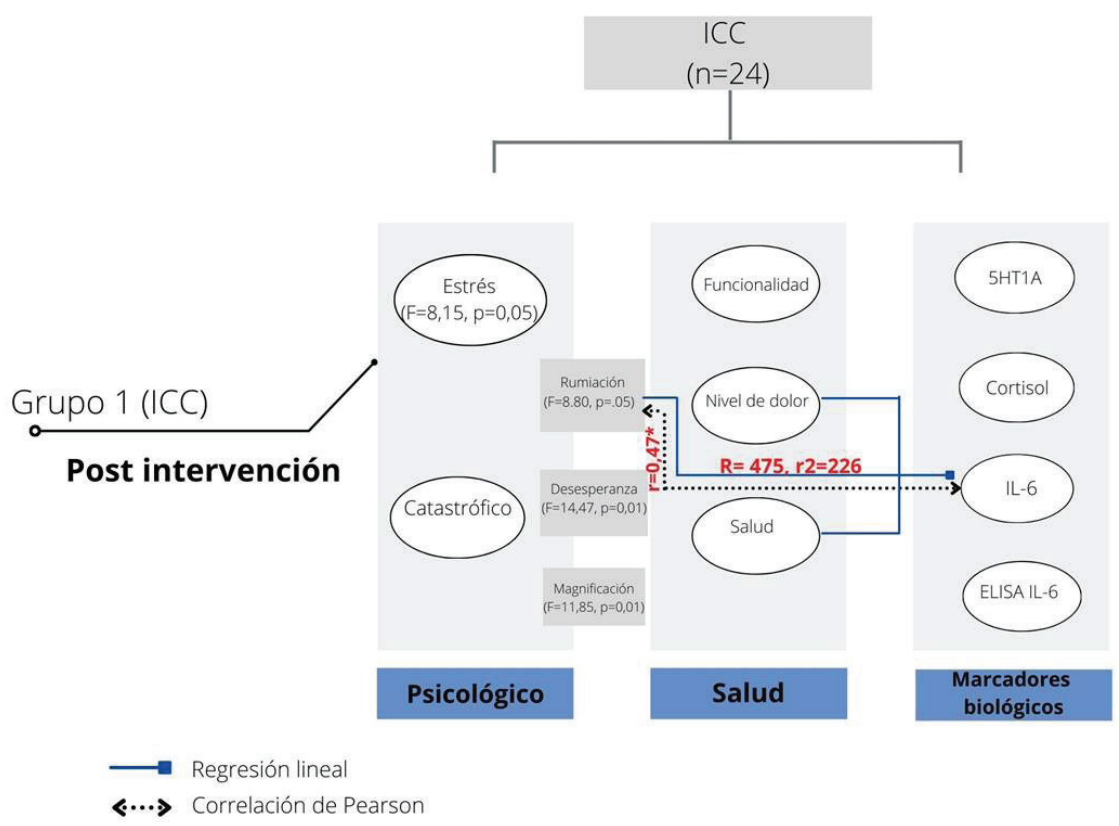

Fig. 2. Resultados de correlaciones y reǵresión lineal en ICC.

La Fig. 3 muestra de forma gráfica los resultados más relevantes de correlaciones y regresión lineal en IPF. Se detectaron tres modelos: el estado de salud predice nivel de dolor; la funcionalidad como predictor del nivel de dolor y el estado de salud como predictor de la expresión del gen del receptor de serotonina (5HT1A). Como se puede observar, variables psicológicas, así como modelos de salud se explican por la expresión génica de IL-6 y del receptor de serotonina (5HT1A).

\section{DISCUSIÓN}

Esta investigación tuvo como objetivo evaluar el impacto de dos intervenciones psicológicas en la modulación de pensamientos catastróficos, estrés percibido y dolor, relacionados con niveles séricos de cortisol e IL-6 y expresión génica de IL-6 y 5 HT1A, en mujeres con FM.

\section{ICC}

Los resultados indican que el grupo de ICC obtuvo una reducción de puntajes fina- 


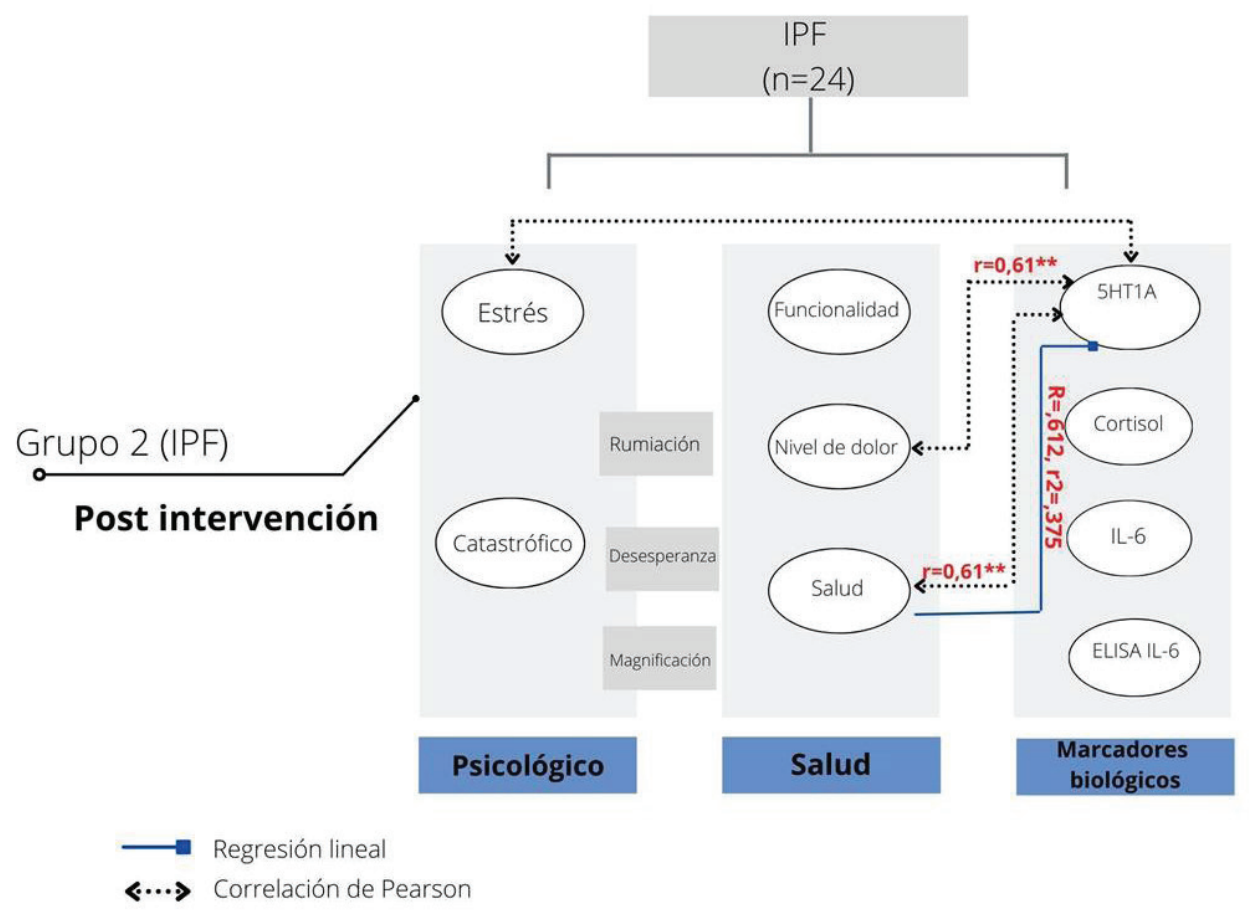

Fig. 3. Resultados de correlaciones y regresión lineal en IPF.

les en percepción de estrés, y catastrofismo en sus tres subescalas: rumiación, maǵnificación y desesperanza; correlacionada con nivel de dolor, percepción de estado de salud y funcionamiento. Estos se asocian a resultados en cortisol en ICG.

La ICG tiene como finalidad la modificación de pensamientos desadaptativos, para lo cual utiliza técnicas específicas y tiene un impacto conductual. Los resultados a partir de la aplicación de ICG sugieren que existe una asociación entre los pensamientos catastróficos y el estrés, lo que propicia una respuesta de sintomatología clínica como dolor y en la escala de funcionalidad física, así como la modificación de la respuesta biológica ante los niveles séricos de cortisol y expresión génica de IL-6. En este sentido, las pacientes a lo largo de esta intervención refirieron que el cambio del pensamiento catastrófico a uno más funcional y adaptativo, les permitió modificar la percepción negativa que se presentaba respecto a su estado de salud, donde se evidenciaba mayor prevalen- cia de desesperanza y maǵnificación basal. Además, funcionalmente adquirieron las herramientas para conocer su sintomatología y hacer actividades físicas de baja intensidad con mayor frecuencia. Estas acciones funcionales les permitieron reducir pensamientos rumiativos y, por lo tanto, el dolor disminuyó significativamente.

\section{Expresión Génica y Niveles Séricos de Cortisol e IL-6}

La expresión génica de IL-6 pre-intervención mostró niveles elevados para ambos grupos, sin embargoo, se observó una disminución de expresión génica post-tratamiento en el grupo ICC, mientras que los niveles de proteína (ELISA IL-6) estaban disminuidos; esta diferencia puede ser atribuible a cambios post-traduccionales que ocurren cuando el ARNm es traducido a proteínas. Estos resultados concuerdan con lo reportado por Clauw (14), Geiss y col. (15) y Rodriguez- Pintó y col. (16), quienes reportaron que en FM existe aumento de niveles séricos de citocinas 
proinflamatorias como la IL-6. Al disminuir el estrés, los pensamientos catastróficos y el dolor, se reduce la expresión génica de IL-6. De igual forma, Lazaridou y col. (10) reportaron en un estudio transversal con 48 pacientes, una correlación de los niveles de IL-6 con factores psicológicos, y concluyeron que el dolor crónico y el catastrofismo se asocian con respuestas elevadas de IL-6.

Sin embargo, en el presente estudio se encontraron cambios positivos en expresión génica, posteriores a la intervención; mientras que en la cuantificación de niveles de proteína no se observaron diferencias significativas entre las concentraciones basales y finales. Estos resultados pueden atribuirse a cambios postranscripcionales, ya que mientras el ARNm que codifica para IL-6 dura tan solo unos minutos, la proteína puede permanecer activa un máximo de 29 horas (38). Estas características muestran un panorama distinto en la intervención de la FM y sugieren explorar nuevas vías en el estudio de este padecimiento.

Ahora bien, Leung (17), encontró que el catastrofismo no sólo se asocia a las alteraciones de IL-6, sino también a una disminución de cortisol. Vachon-Presseau y col. (39), concluyeron que los ritmos de cortisol eran predictores de dolor. Una respuesta de estrés prolongada puede mantener una disfunción en los niveles de cortisol y dolor, el agotamiento y la hiperexcitabilidad provocan una disminución en la secreción de cortisol. Es decir, la hiperactividad simpática mantenida a largo plazo provoca degradación en el sistema, dificultando que el cuerpo regrese a un estado de reposo (1). La hiperactividad simpática es una de las características principales en FM; existe una causa biológica generada por el estrés, que refieren las pacientes al hacer sus actividades de manera excesiva y presentar pensamientos catastróficos, estos fueron factores que se trabajaron en el grupo. Por lo tanto, las investigaciones previas y lo encontrado en el presente trabajo, posiblemente confirman la relación de estas variables: El estado inflamatorio está acompañado de una respuesta de estrés alterada en pacientes con FM.

Los resultados del grupo ICG mostraron que esta intervención puede ser potencial para el tratamiento psicológico en FM. Las correlaciones y las regresiones lineales encontradas en este estudio señalan que el catastrofismo (rumiación) y la expresión génica de IL-6, son modelos predictores en FM, es decir, si se interviene en la reducción del catastrofismo (rumiación), los niveles de IL-6 probablemente disminuirán.

\section{IPF}

En lo que respecta a la IPF, se encontró reducción de puntajes finales en percepción de estrés y catastrofismo, aunque este no fue significativo en comparación con ICG. La IPF se basó específicamente en la expresión emocional y técnicas de relajación como la respiración y control autógeno, esto con el objetivo de generar un cambio en el sistema autónomo por medio del nervio vaǵo, lo que coincide con las investigaciones de Busch y col. (36) e Iwabe y col. (40), las cuales reportaron que la respiración y las técnicas de relajación inducen cambios en el sistema autónomo, sobre todo en la actividad simpática y el estrés.

Las pacientes a partir del entrenamiento de respiración diafragmática y control autógeno, refirieron disminución de estrés y nivel de dolor. Las actividades de expresión emocional por medio de la escritura y reconocimiento facial, les dieron las herramientas para detectar en qué momentos de estrés presentaban dolor y pudieron incorporar la respiración como un recurso de control fisiológico. La IPF parece mejorar la parte fisiológica y el estrés de manera conjunta, reduciendo los niveles de dolor, lo cual se comprobó con los resultados post tratamiento en esta investigación, ya que indican que hay un cambio significativo en el nivel de dolor, estado de salud y funcionalidad. Correlacionando el estado de salud con el nivel de dolor y estrés, se constata que el entrenamiento de respiración reduce sín- 
tomas de FM (35). En cuanto a la respuesta biológica, la IPF no logró inducir efectos relacionados con la reactividad de cortisol y la expresión génica de IL-6, ya que hubo aumento de ambos. Sin embargoo, sí mostró modificación respecto a la expresión génica del receptor de serotonina 5HT1A. Lo que posiblemente indique que al utilizar únicamente técnicas de relajación, que incluyen la respiración diafragmática, se logre modificar la percepción del estímulo que produce dolor. Esta relación inversa entre los niveles plasmáticos de cortisol y la expresión génica del receptor 5HT1A reportados en el presente estudio, concuerda con lo descrito por Steinberǵ, y col. (41), quienes mencionaron que la disfunción del eje HPA está directamente relacionado con la disfunción serotoninérǵica, específicamente con los cambios en el receptor 5HT1A. Debido a que el cortisol inhibe la síntesis L-triptófano, disminuye su disponibilidad para la síntesis de serotonina $(41,42)$.

La correlación negativa entre el cortisol plasmático en reposo y la expresión del receptor de serotonina 5HT1A, explicaría los cambios post-tratamiento observados en el cortisol y el receptor de serotonina en ambas intervenciones; es decir, mientras aumentaba uno, los niveles del otro disminuían. Además, se ha reportado que en mujeres con FM, que concentraciones más altas de cortisol están relacionadas con menor intensidad de dolor y, en consecuencia, las concentraciones más bajas de cortisol se han relacionado con mayores niveles de dolor (43). Por lo que consideramos que esta es un área que debe investigarse a profundidad.

Las limitantes del estudio residen en el tamaño de la muestra, y en el criterio de exclusión de depresión. Por lo que en próximas investigaciones, sugerimos aumentar el tamaño de la muestra, e incluir sujetos con criterios clínicos de depresión, con la finalidad de confirmar con mayor precisión el efecto específico de las intervenciones. Así mismo, sugerimos explorar otras vías moleculares que pudiesen estar involucradas en la etiopatogenia de la FM, en lo referente al sistema inmune de las pacientes y a la relación con vías de estrés, para tener una amplia visión de aquellos factores predictores.

En conclusión, este estudio sugiere que la modulación del pensamiento catastrófico tiene influencia significativa a nivel cognitivo, emocional y en la expresión génica de IL-6. Debido a que se obtuvo correlación positiva entre el catastrofismo (rumiación) y la expresión génica de IL-6; incluyendo al hallazgo del modelo de rumiación como predictor de la expresión génica de IL-6. Lo cual indica que posiblemente existe un proceso proinflamatorio en FM y al mismo tiempo este puede ser tratado empleando la ICC.

Por el contrario, los resultados de la modulación de los niveles séricos de IL-6, abren la interrogante sobre mecanismos alternos que pudieran estar influyendo en los procesos inflamatorios, al presentarse una vida media corta la expresión génica comparada con la proteína.

Finalmente, en ambas intervenciones disminuyó la percepción del dolor y el estrés. Sin embargó, la ICC mostró mayor efectividad en las variables psicológicas y metabólicas; por lo tanto, la ICC sería una propuesta de tratamiento con mayor eficacia para mujeres con FM.

\section{REFERENCIAS}

1. Martinez-Lavin M. Fibromyalgia: when distress becomes (Un) sympathetic pain. Pain Res Treat 2012;2012: 1-6.Article ID 981565.

2. Barrera Villalpando MI, Cortes Sotres JF, Mendieta Cabrera D, Guerrero León D. Reduction of the parasympathetic influx in fibromyalgia: Its relationship with psychiatry in a national referral specialized center. Salud Ment 2015;38(2):123-128.

3. Behm FG, Gavin IM, Karpenko O, Lindgren V, Gaitonde S, Gashkoff PA, Gillis BS. Unique immunologic patterns in fibromyalgia. BMC Clin Pathol 2012;12(25):1-7.

4. Kosek E, Altawil R, Kadetoff D, Finn A, Westman M, Le Maître E, Andersson M,

Vol. 62(2): 140 - 158, 2021 
Jensen-Urstad M, Lampa J. Evidence of different mediators of central inflammation in dysfunctional and inflammatory pain-interleukin-8 in fibromyalgia and interleukin-1 $\beta$ in rheumatoid arthritis. J Neuroimmunol 2015;280:49-55.

5. Mendieta D, Luz D, Cruz-Aguilera D, Barrera-Villalpando MI, Becerril-Villanueva E, Arreola R, Hernández-Ferreira E, Pérez-Tapia SM, Pérez-Sánchez G, Garcés-Alvarez ME, Aguirre-Cruz L, Velasco-Velázquez MA, Pavón L. IL-8 and IL-6 primarily mediate the inflammatory response in fibromyalgia patients. J Neuroimmunol 2016;290:22-25. Available from: http://dx.doi.org/10.1016/j.jneuroim. 2015.11.011

6. Hannibal KE, Bishop MD. Chronic stress, cortisol dysfunction, and pain : A psychoneuroendocrine rationale for stress management in pain rehabilitation. Phys Ther 2014;94:1816-1825.

7. Barceló-Martinez E, Gelves-Ospina M, Navarro Lechuga E, Allegri RF, OrozcoAcosta E, Benitez-Agudelo JC, León-Jacobus A, Román NF. Serum cortisol levels and neuropsychological impairments in patients diagnosed with fibromyalgia. Actas Españolas Psiquiatr 2018;46(1):1-11.

8. Taylor AG, Adelstein KE, Fischer-White TG, Murugesan M, Anderson JG. Perspectives on living with fibromyalgía. Glob Qual Nurs Res 2016;3:1-13.

9. Sturgeon JA, Zautra AJ. State and trait pain catastrophizing and emotional health in rheumatoid arthritis. Ann Behav Med 2014;45(1):69-77.

10. Lazaridou A, Martel MO, Cahalan CM, Franceschelli O, Campbell CM, Haythornthwaite JA, Smith M, Riley J, Edwards RR. The impact of anxiety and catastrophizing on interleukin- 6 responses to acute painful stress. J Pain Res 2018;11:637-647.

11. Burri A, Ogata S, Rice D, Williams F. Pain catastrophizing, neuroticism, fear of pain, and anxiety: defining the genetic and environmental factors in a sample of female twins. PLoS One 2018;13(3):1-15.

12. Morris LD, Louw QA, Grimmer KA, Meintjes E. Targeting pain catastrophization in patients with fibromyalgia using virtual re- ality exposure therapy : a proof-of-concept study. J Phys Ther Sei 2015;27:3461-3467.

13. Sharma S, Thibault P, Abbott JH, Jensen MP. Clinimetric properties of the Nepali version of the Pain Catastrophizing Scale in individuals with chronic pain. J Pain Res 2018;11:265-276.

14. Clauw DJ. Fibromyalgia. A clinical review. JAMA 2014;311(15):1547-1555.

15. Geiss A, Rohleder N, Anton F. Evidence for an association between an enhanced reactivity of interleukin-6 levels and reduced glucocorticoid sensitivity in patients with fibromyalgia. Psychoneuroendocrinology 2012;37(5):671-684. Available from: http://dx.doi.org/10.1016/j.psyneuen. 2011.07.021.

16. Rodriguez-Pintó I, Agmon-levin N, Howard A, Shoenfeld Y. Fibromyalgia and cytokines. Immunol Lett 2014;161(2):200-203. Available from: http://dx.doi.org/10.1016/j. imlet.2014.01.009.

17. Leung L. Pain catastrophizing : an updated review. Indian J Psychol Med 2012; 34(3):204-217.

18. Shivers KY, Amador N, Abrams L, Hunter $\mathrm{D}$, Jenab S, Quiñones-Jenab V. Estrogen alters baseline and inflammatory-induced cytokine levels independent from hypothalamic-pituitary-adrenal axis activity. Cytokine 2015;72(2):121-129.

19. Park DJ, Kang JH, Yim YR, Kim JE, Lee JW, Lee KE, Wen L, Kim TJ, Park YW, Lee SS. Exploring genetic susceptibility to fibromyalgia. Chonnam Med J 2015;51(2):58-65.

20. Kosek E, Martinsen S, Gerdle B, Mannerkorpi K, Löfgren M, Bileviciute-ljungar I, Fransson $\mathrm{P}$, Schalling $\mathrm{M}$, Ingvar M, Emberg M, Jensen KB. The translocator protein gene is associated with symptom severity and cerebral pain processing in fibromyalgia. Brain Behav Immun 2016;58:218-227. Available from: http:// dx.doi.org/10.1016/j.bbi.2016.07.150.

21. Köllner V, Häuser W, Klimezyk K, KühnBecker H, Settan M, Weigl M, Bernardy K. Psychotherapie von patienten mit fibromyalgiesyndrom. Schmerz 2012;26:291-296.

22. Lami MJ, Martínez MP, Sánchez AI. Systematic review of psychological treatment 
in fibromyalgia. Curr Pain Headache Rep 2013;17(7):345-358.

23. Glasgow A, Stone TM, Kingsley JD. Resistance rxercise training on disease impact, pain catastrophizing and autonomic modulation in women with fibromyalgia. Int Journal Exere Sci 2017;10(8):1184-1195.

24. Schmidt JE, Brien TGO, Hooten WM, Joyner MJ, Johnson BD. The effects of slow-paced versus mechanically assisted breathing on autonomic function in fibromyalgia patients. J Pain Res 2017;10:2761-2768.

25. Häuser W, Ablin J, Perrot S, Fitzcharles MA. Management of fibromyalgia: key messages from recent evidence-based guidelines. Polish Arch Intern Med 2017;127(1):47-56.

26. Thieme K, Mathys M, Turk DC. Evidenced-based guidelines on the treatment of fibromyalgia patients: are they consistent and if not, why not? Have effective psychological treatments been overlooked? J Pain

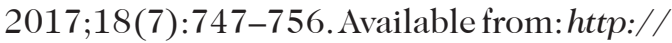
dx.doi.org/10.1016/j.jpain.2016.12.006

27. Giusti EM, Castelnuovo G, Molinari E. Differences in multidisciplinary and interdisciplinary treatment programs for fibromyalgia: a mapping review. Pain Res Manag 2017;2017:7261468. doi: 10. $1155 / 2017 / 7261468$.

28. Lumley MA, Schubiner H, Lockhart NA, Kidwell KM, Harte SE, Clauw DJ, Williams DA. Emotional awareness and expression therapy, cognitive-behavioral therapy, and education for fibromyalgia: a cluster-randomized controlled trial. Pain 2017;158(12):2354-2363.

29. MeCrae CS, Williams J, Roditi D, Anderson R, Mundt JM, Miller MB, Curtis AF, Waxenberg LB, Staud R, Berry RB, Robinson ME. Cognitive behavioral treatments for insomnia and pain in adults with comorbid chronic insomnia and fibromyalgia: clinical outcomes from the SPIN randomized controlled trial. Sleep 2019;42(3):1-15.

30. Porges SW. The polyvagal perspective. Biol Psychol 2007;74(2):116-143.

31. Meeus M, Nijs J, Hermans L, Goubert D, Calders P. The role of mitochondrial dysfunctions due to oxidative and nitrosative stress in the chronic pain or chronic fatigue syndromes and fibromyalgia patients: Peripheral and central mechanisms as the- rapeutic targets? Expert Opin Ther Targets 2013;17(9):1081-1089.

32. Kang JH, Kim JK, Hong SH, Lee CH, Choi BY. Heart rate variability for quantification of autonomic dysfunction in fibromyalgia. Ann Rehabil Med 2016;40(2):301-309.

33. Garrido M, Castaño MY, Biehl-Printes C, Gomez MA, Branco JC, Tomas-Carus P, Rodriguez AB. Effects of a respiratory functional training program on pain and sleep quality in patients with fibromyalgia: A pilot study. Complement Ther Clin Pract 2017;28:116-121. Available from: http:// dx.doi.org/10.1016/j.ctcp.2017.05.013

34. Jafari H, Courtois I, Van Den Bergh O, Vlaeyen JWS, Van Diest I. Pain and respiration: A systematic review. Pain 2017;158(6):9951006.

35. Katzman M, Vermani M, Gerbarg PL, Brown RP, Iorio C, Davis M, Cameron C, Tsirgielis D. A multicomponent yogabased, breath intervention program as an adjunctive treatment in patients suffering from generalized anxiety disorder with or without comorbidities. Int $\mathrm{J}$ Yoga 2012;5(1):57-65. Available from: http:// wrww.ijoy.org.in/text.asp? 2012/5/1/57/ 91716

36. Busch V, Magerl W, Kern U, Haas J, Hajak G, Eichhammer P. The effect of deep and slow breathing on pain perception, autonomic activity, and mood processing - An experimental Study. Pain Med (United States) 2012;13:215-228.

37. Tomas-Carus $\mathrm{P}$, Garrido M, Branco JC, Castaño MY, Gómez MÁ, Biehl-Printes C. Non-supervised breathing exercise reģimen in women with fibromyalǵia: A quasiexperimental exploratory study. Complement Ther Clin Pract 2019;35:170-176. Available from: https://doi.org/10.1016/j. ctcp.2019.02.006

38. Kuribayashi T. Elimination half-lives of interleukin-6 and cytokine-induced neutrophil chemoattractant-1 synthesized in response to inflammatory stimulation in rats. Lab Anim Res 2018: 34(2), 80-83. Available from: https://doi.org/10.5625/ lar.2018.34.2.80.

39. Vachon-Presseau E, Martel MO, Roy M, Caron E, Albouy G, Marin MF, Plante I, Sullivan MJ, Lupien SJ, Rainville P. Acute

Vol. 62(2): 140 - 158, 2021 
stress contributes to individual differences in pain and pain-related brain activity in healthy and chronic pain patients. J Neurosei 2013;33(16):6826-6833.

40. Iwabe T, Ozaki I, Hashizume A. The respiratory cycle modulates brain potentials, sympathetic activity, and subjective pain sensation induced by noxious stimulation. Neurosci Res 2014;84:47-59. Available from: http://dx.doi.org $/ 10.1016 / j$.neures. 2014.03.003

41. Steinberg LJ, Rubin-Falcone H, Galfalvy HC, Kaufman J, Miller JM, Sublette ME, Cooper TB, Min E, Keilp JG, Stanley BH, Oquenda MA, Ogden RT, Mann JJ. Cortisol stress response and in vivo pet imaging of human brain serotonin 1a receptor binding. Int $\mathrm{J}$ Neuropsychopharmacol 2019;22(5):329-338.
42. Badawy AA, Morgan CJ, Lovett JW, Bradley DM, Thomas R. Decrease in circulating tryptophan availability to the brain after acute ethanol consumption by normal volunteers: implications for alcohol-induced aggressive behaviour and depression. Pharmacopsychiatry 1995;28:93-97.

43. Úbeda-D'Ocasar E, Jiménez Díaz-Benito V, Gallego-Sendarrubias GM, Valero-Calero JA, Vicario-Merino JA, Vicario-Merino A, Hervás-Pérez JP. Pain and cortisol in patients with fibromyalgia: systematic review and meta-analysis. Diagnostics (Basel) 2020;10(11):922. doi: 10.3390/diaǵnostics10110922. 